Revista Iberoamericana, Vol. LXVIII, Núm. 201, Octubre-Diciembre 2002, 1091-1109

\title{
ESTUDIOS SUBALTERNOS Y TIEMPO INSURGENTE: MEMORIAS MAYA-TZELTAL DE LA REBELION DE 1712 EN CHIAPAS ${ }^{1}$
}

\author{
POR
}

Luis Fernando Restrepo

University of Arkansas

El libro Jwan Lopes, Bats'il ajaw/Juan López, héroe tzeltal (1996) de Domingo Gómez Gutiérrez, un tzeltal de Chilón, Chiapas, compila siete narraciones maya tzeltales sobre un personaje de la rebelión indígena de 1712 en esta región. El presente trabajo se enfoca en estas narraciones, pero hace parte de un estudio más amplio que revisa críticamente lo que se ha escrito sobre esta rebelión desde el siglo XVIII en adelante en diferentes campos como la literatura, la legislación, la historia y la antropología. El punto de partida es un artículo sobre las rebeliones campesinas en la India desarrollado por el historiador Ranajit Guha, uno de los fundadores del colectivo de estudios subalternos. ${ }^{2}$ En “The Prose of Counter-Insurgency”, Guha acertadamente señalaba cómo la historiografía, tanto colonial como nacional, tendía a ver la participación popular en términos negativos;

${ }^{1}$ Este trabajo amplía la ponencia titulada "Unwriting History: Contemporary Oral Narratives on the 1712 Maya Rebellion in Chiapas” presentada en la mesa “Indigenous Literatures and New Social Movements” organizada por Cynthia Steel en el MLA en Chicago, 1999, a quien agradezco enormemente por todo su apoyo. También quiero expresar mi gratitud a quienes leyeron versiones anteriores de este trabajo, entre ellos, Marc Zimmerman y Jan Rus.

${ }^{2}$ Brevemente dicho, los estudios subalternos surgen de un colectivo de historiadores hindúes, encabezado por Ranajit Guha, quienes a partir de la década de los ochenta empezaron a revisar los prejuicios y puntos ciegos de la historiografía de la India. Basados en intelectuales marxistas no ortodoxos como Antonio Gramsci, se resaltó cómo tanto la historiografía colonial como nacional hindú negaban la participación y la conciencia populares. Más adelante, con el ensayo seminal de Gayatri Spivak, “Can the Subaltern Speak?” (publicado inicialmente en 1985 y reelaborado años más tarde en A Critique of Postcolonial Reason) el campo comienza a abordar el posestructuralismo francés y la crítica poscolonial enfrentando una gama de problemáticas mucho más amplia como la construcción, representación y subordinación de sujetos (en términos de étnia, género y clase), territorios y campos del saber, así como la nación y la modernidad. Paralelamente surge en los Estados Unidos un grupo homólogo enfocado en las Américas. Véanse por ejemplo Selected Subaltern Studies (1988) editado por Guha y Spivak, A Subaltern Studies Reader 1986-1995 (1997) editado por Guha y Mapping Subaltern Studies and the Postcolonial (2000) editado por Vinayak Chaturvedi. En relación a las Américas, véanse el número dedicado al tema por la revista Dispositio/ $n$ (1994) y dos recientes volúmenes editados por Ileana Rodríguez, The Latin American Subaltern Studies Reader (2001) y Convergencia de tiempos: estudios subalternos / contextos latinoamericanos, estado, cultura, subalternidad (2001). Este campo si bien ilumina aspectos claves del proceso histórico-social, no es infalible, lo cual ha empezado a problematizar la crítica. Véanse “Teoría, 
es decir, se veían principalmente como un problema de orden público. Igualmente, se tendía a negar su conciencia histórica al presentar los movimientos insurgentes como fenómenos naturales (epidemias, incendios) o bien como respuestas mecánicas a presiones externas (impuestos, sequías, hambrunas). Al analizar los relatos sobre las rebeliones, Guha identifica tres tipos de discursos. El discurso primario sería el de aquellos textos producidos por personas testigos de los acontecimientos (soldados, oficiales, etc.). El discurso secundario es generalmente posterior a los hechos, compila varios relatos primarios y establece relaciones causales. Ambos discursos están escritos desde la lógica del estado y de una manera u otra abogan por la intervención estatal y la restauración del orden. Por esto, Guha los denomina como "prosa contra-insurgente”. El tercer tipo de discurso se caracteriza precisamente por tratar de romper con la "prosa contra-insurgente" y presentar la rebelión desde el punto de vista de los insurgentes (72). Al omitir la religiosidad como un elemento clave en la conciencia histórica popular, argumenta Guha, el discurso terciario no logra captar adecuadamente la experiencia subalterna de la rebelión. En un estudio más reciente, Dipesh Chakrabarty, otro historiador del colectivo hindú, lleva este último punto incluso mucho más allá, señalando los límites de la historiografía occidental secular para narrar historias donde intervienen fuerzas divinas, sin que esto permita clasificar dichos movimientos como "arcaicos” o prepolíticos: "Instead of being an anachronism in a modernizing colonial world, the peasant was a real contemporary of colonialism, a fundamental part of the modernity that colonial rule brought to India” $(13,103) .^{3}$

Tomando bien en cuenta el contexto colonial americano (y sus divergencias con la experiencia asiática), el estudio de Guha nos puede ayudar a comprender la amplia gama

política, subalternidad y poscolonialidad” (1999), entrevista a Aijaz Ahmad, "The Promise and Dilemma of Subaltern Studies: Perspectives from Latin American History” (1994) de Florencia Mallón, "The Fantasies of Cultural Exchange in Latin American Subaltern Studies" (1996) de Gareth Williams y "El boom del subalterno" (1997) de Mabel Moraña.

${ }^{3}$ Chakrabarty a pesar de que cuestiona los presupuestos del historicismo occidental, el macro relato del progreso, se limita quizás demasiado a una noción bastante ortodoxa de la práctica histórica, al ámbito académico. "Uno no escribe para el subalterno”, dice entre paréntesis en la página 89! También hay cierto paternalismo al decir que los académicos deben ayudar al subalterno a plantear propuestas en términos seculares: "One cannot argue with modern bureaucracies and other instruments of governmentality without recourse to the secular time and narratives of history and sociology. The subaltern classes need this knowledge in order to fight their battles of social justice. It would therefore be unethical not to make historical consciousness available to everybody, in particular the subaltern classes” (86). Este paternalismo, como bien señala Rabasa en su epílogo a Writing Violence (2000), es problemático pues asume incapaces a los subalternos de moverse en múltiples niveles (277). Se suma también a una problemática concepción del subalterno en términos negativos al verlo por "fuera” del marco representacional. "Negativity bars subalterns from turning the Enlightenment against its own biases, by arguing the human right to live and express oneself in terms of the times of the gods” (277). En síntesis, la dualidad tiempo secular-tiempo divino que deja intacta Chakrabarty, tiene su clara raigambre ilustrada. El discurso ilustrado es precisamente excluyente de lo que no cabe en la concepción secular del mundo. Pero este mundo, argumenta Rabasa, está concebido, simultámente, de múltiples formas que estallan la homogeneidad del tiempo secular (278). Como veremos, esta pluralidad temporal es clave para comprender las historias mayas discutidas en el presente artículo. 
de relatos que ha generado la rebelión maya de 1712, organizada en torno al culto de la Virgen. Una vasta documentación colonial existente hoy en el Archivo de Indias, México y Guatemala parece confirmar lo expuesto por Guha. ${ }^{4}$ Sin embargo, las historias de Juan López, pertenecientes a la tradición oral y transcritas casi trescientos años después de la rebelión, no caben en ninguna de las categorías definidas por el historiador hindú. Estas narraciones bien podrían considerarse un cuarto discurso, provenientes de los descendientes del grupo insurgente mismo. Se trata de una memoria subalterna, aunque no puede verse ingenuamente como la voz "nativa", esencializando al subalterno, sino tomando en cuenta los contextos políticos y discursivos que enmarcan su voz, como bien lo ha señalado Gayatri Spivak (1999). Considerando este último punto, comenzaremos examinando los contextos históricos en los cuales son transcritas las historias orales mayas sobre la rebelión chiapaneca colonial. Luego veremos en las historias mismas el tipo de memoria que se plasma a través de Juan López, una compleja figura heroica, mítica e histórica a la vez, la cual revela la existencia de una pluritemporalidad que ha tratado de suprimir el discurso ilustrado.

\section{Historias LOCALES EN CONTEXTOS NEOCOLONIALES}

Las historias tzeltales sobre Juan López fueron compiladas y transcritas en un contexto que no puede desconocerse. En 1996 estas historias aparecieron en edición bilingüe en la colección Letras Mayas Contemporáneas, dirigida por Carlos Montemayor, quien hizo también las traducciones al castellano. Esta colección fue coauspiciada por el Instituto Nacional Indigenista (INI), la Fundación Rockefeller, la Unidad de Escritores Mayas-Zoques y el colectivo de escritores indígena Sna Jtz'ibajom (La casa del escritor). El Sna, fundado en San Cristóbal en 1983, ha venido realizando numerosos proyectos de revitalización de la cultura y memoria mayas. Tienen grupos de lectura y discusión de textos como el Popol Vuh y otros textos mayas, talleres de escritura, campañas de alfabetización en lenguas maya, además de la producción de videos, teatro popular y programas de radio (Pérez Hernández 100; Steele; Frischmann). Los participantes del colectivo Sna han recibido apoyo de diferentes agencias e instituciones gubernamentales y ONGs. Entre ellas se encuentran el Instituto Chiapaneco de Cultura, Mex-Fam, la Fundación Kellog, Smithsonian Institution, XEVS Radio Margaritas, la UNAM, etc. Su grupo de teatro ha viajado por todo México y al exterior. Otros de sus participantes han viajado a otras regiones mayas para encuentros y proyectos con otros grupos indígenas, activistas políticos, escritores y académicos.

Es también importante tener en cuenta la historia de Chiapas en el contexto de las políticas culturales mexicanas y el auge de los “estudios de área” en las universidades norteamericanas a partir de la posguerra. Desde la conferencia indigenista de Patzcuaro (1940), Chiapas se convirtió en el proyecto piloto del Instituto Nacional Indigenista (INI),

\footnotetext{
${ }^{4}$ La documentación oficial principal se encuentra reunida en cuatro legajos en el Archivo General de Indias (AGI), Audiencia de Guatemala 293-296. Una documentación adicional se encuentra en diversos archivos en México y Guatemala. Véanse Juan Pedro Viqueira (1997) y Kevin Gosner (1992).
} 
fundado en 1951. Esta región ha sido escogida para numerosos proyectos antropológicos como los estudios de la Escuela Nacional de Antropología e Historia a partir de 1940, el Proyecto Harvard (1957-72) dirigido por Evon Z. Vogt y el "Man-in-Nature Project” de la Universidad de Chicago en la década de $1960 .{ }^{5}$ El colectivo del Sna ha trabajado en conjunto con antropólogos como Robert Laughlin del Smithsonian Institution. Adicionalmente, Chiapas también fue clave para probar las nuevas políticas culturales de la Iglesia Católica tras el Vaticano Segundo (1965) y la Conferencia Episcopal en Medellín (1968) donde se planteó la necesidad de elaborar un discurso cristiano que apelara a los marginados, los pobres, las mujeres, las minorías y los indígenas (Marzal 140), origen de las comunidades eclesiásticas de base (CEB). Toda esta información nos es preciso tener en cuenta para no ver ingenuamente las historias en cuestión y poder considerar estas voces mayas en un marco histórico mucho más amplio que tenga en cuenta las complejas relaciones entre lo local y lo global, a costo quizás de una discusión más profunda de los textos mismos.

Lo que quiero resaltar es que se trata sin duda de historias que no surgen en un vacío y que es preciso historizar la producción discursiva de Chiapas. Nos encontramos ante el trabajo intelectual de actores mayas contemporáneos involucrados en una compleja relación con la modernidad. Interpelados por las políticas nacionales y neocoloniales para preservar y promover las culturas indígenas, estos intelectuales mayas están redefiniéndose a sí mismo, negociando y transformando sus "tradiciones” en los complejos mercados culturales locales, nacionales y transnacionales (Steele). Siento que es preciso ver estas narraciones mayas en estos contextos, pues sin ellos sería fácil convertirlas en objetos de museo para ser coleccionados y preservados, en un gesto propulsado más por la nostalgia o la fascinación con el objeto mismo, sin tomar en cuenta los agentes sociales involucrados, sus relaciones con dichos productos simbólicos y las instituciones que circunscriben y median su producción, circulación y consumo. Esto es similar a lo que sucede con la valoración tradicional de las artesanías, concebidas como populares, premodernas y ajenas a la innovación discutidas por Néstor García Canclini en Culturas híbridas, cuando en realidad tienen una relación mucho más dinámica con los mercados modernos capitalistas (198-99). ${ }^{6}$ La propia factura de las historias sobre Juan López nos revela esta situación intersticial.

${ }^{5}$ Sobre el proyecto de Chiapas de Harvard, véase "Rereading Tzotzil Ethnography: Recent Scholarship from Chiapas, Mexico" de Jan Rus.

${ }^{6}$ Véase también Rowe y Schelling en Memory and Modernity (67). Es revelador que dos años antes de la aparición del "Founding Statement” del Latin American Subaltern Studies Group en Boundary 2, este libro de Rowe y Schelling sobre las culturas populares presenta ya un trabajo que busca rescribir la historia de las Américas desde una perspectiva subalterna en términos claramente paralelos a los planteados por Guha y el colectivo hindú, sin basarse en ellos, pero sí tomando de Gramsci y los estudios culturales. Si bien, en América Latina como en otras partes del mundo, desde mediados del siglo XX surgieron trabajos que buscaban escribir la historia desde "abajo”, el límite de dichos estudios era presuponer unos sujetos a priori sobre los cuales se habría impuesto la colonización europea. El salto paradigmático tanto de los estudios culturales como subalternos, se da en cuanto se conciben los sujetos al interior de la historia misma y a través de las relaciones de poder que los configuran, en un proceso vivido, nunca fijo, como bien lo plantea Jesús Martín Barbero: "Está, en primer lugar, el concepto de hegemonía elaborado por Gramsci, haciendo posible 
Entre la oralidad y la escritura: las siete historias y la "versión conjuntada”

Las historias recopiladas y transcritas por Gómez Gutiérrez son siete narraciones breves de cuatro a catorce páginas de extensión. Son relatos de procedencia claramente oral, aunque han sido reducidos a la monotonía de la letra, sin ninguna marca respecto a la entonación, los gestos, silencios, repeticiones y otros rasgos claves en la comunicación oral. Son relatos, en todo caso, que distan mucho de las etnoficciones que describe Martin Lienhard y otros textos similares recientes que tratan esta rebelión como la novela Jovel, serenata de la gente menuda (1992) de Heberto Morales. ${ }^{7}$ No obstante, no puede ignorarse históricamente por qué se traducen y compilan testimonios y otros discursos similares. Lienhard señala las implicaciones de la edición de textos como los que se discuten en este ensayo:

Ahora, de por sí la publicación de testimonios populares contemporáneos tampoco garantiza la irrupción de cosmovisiones realmente "alternativas" en la esfera letrada. Siendo el editor (= compilador) quien elige y "formatea" las voces populares que juzga dignas de acceder a la imprenta, los testimonios publicados se inscriben por lo general en los proyectos editoriales concebidos en y a partir de los intereses de la ciudad letrada o el sector hegemónico. ("Voces” 798)

Gómez Gutiérrez compila las historias en un relato final que titula “versión conjuntada”. Este texto del compilador no coteja sistemáticamente las versiones sino que simplemente toma elementos de una historia y otra, sin explicitar los parámetros que rigen dicha selección, un proceso que en cierta manera sigue más la lógica de la memoria oral que de la escritura, donde el relato es reelaborado por cada narrador.

Las historias sobre Juan López están primordialmente escritas para un “nosotros” tzeltal. Es más que todo un discurso para consumo interno que un discurso minoritario. En este último, se proyecta estratégicamente lo étnico en el espacio público hegemónico como una movida política contestataria (el caso de Rigoberta Mechú, por ejemplo). ${ }^{8}$ Sin embargo, mirando más a fondo vemos que el esquema interno/externo es inadecuado al considerar la situación liminal de estas narraciones solicitadas a los ancianos por un intelectual indígena, Gómez Gutiérrez, quien también habla a un “nosotros” tzeltales pero que apoya su relato con una bibliografía académica y citando el Popol Vuh, considerado aquí como una "verdadera historia del pasado" (117). ${ }^{9}$

Como productos culturales estas historias pueden ser decodificadas a través de un marco cultural compartido por una comunidad interpretativa. Sin embargo, al pertenecer a una "zona de contacto", esto es mucho más complejo porque tenemos por lo menos dos

pensar el proceso de dominación social ya no como imposición desde un exterior y sin sujetos, sino como un proceso en el que una clase hegemoniza en la medida en que representa intereses que también reconocen de alguna manera como suyos las clases subalternas” (84-85).

${ }^{7}$ Esta novela cuenta la historia de Ciudad Real desde una perspectiva mestiza.

${ }^{8}$ Me baso en la definición del discurso minoritario discutida por David Lloyd (222).

${ }^{9}$ Gómez Gutiérrez en su bibliografía cita a Víctor Esponda Jimeno, Stefano Varese, Adrián Recinos, Jacinto Arias Pérez y Prudencio Moscoso (131). 
códigos culturales, en relación asimétrica, diglósica, que no están completamente aislados, donde no hay ni un mestizaje armónico, ni una transculturación al código dominante (el camino de Arguedas), ni una hibridización unidimensional. Hay más bien yuxtaposiciones, confluencias, divergencias, contraposiciones y ambivalencias. Por lo tanto es preciso tomar en cuenta tanto la cultura maya como la cultura occidental para leer competentemente estos textos. Es decir, el paradigma de la ciudad letrada es inadecuado para leer estas historias que distan mucho de las novelas indigenistas, por una parte, y, por otra, de los informes y declaraciones que encontramos en el Archivo de Sevilla. ${ }^{10}$ También es preciso tener en cuenta que las comunidades interpretativas no son entes cerrados herméticamente, sino que tienen fronteras bastante porosas y ambiguas, como señala Chatterjee, mucho más de lo que el Estado cuantificador quisiera (223).

Es entonces en un conflictivo intersticio donde surgen e intervienen estos relatos mayas, en el marco de una violenta interpelación (neo)colonial en el cual "el informante nativo” (Gómez Gutiérrez y los siete ancianos) es interpelado y puede hablar. Como bien ha argumentado Spivak, es preciso considerar los límites (la no transparencia del sujeto subalterno) y los contextos en los cuales hablan el subalterno y el informante nativo. ${ }^{11}$

${ }^{10}$ Es lo que sucede con las narraciones indígenas sobre la rebelión de Tupac Amarú que discute Beverley. Unas, orientadas hacia el discurso hegemónico, articulan un sujeto autobiográfico en términos europeos. Otras en cambio articulan una historia mítica, colectiva y ritual (52).

11 La gran dificultad de la representación del subalterno en nuestro discurso académico es precisamente el eje central del reciente libro de John Beverley Subalternity and Representation (1999): "Is it really possible to represent the subaltern from the position of either the disciplinary historian or the literary critic — that is, from the institutional position of the dominant culture?”(20). Beverley responde un sí matizado que considera los estudios subalternos como un "espacio experimental” el cual busca una coalición política de diversos sectores, incluyendo el académico en pos de la igualdad social, la liberación y la reimaginación del estado-nacional en forma más radical que el multiculturalismo del humanismo (neo) liberal (23). Es decir, pese a que Beverley critica a Mallón de retener el macro relato de la nación, él mismo lo ve como clave en un proyecto emancipador, si se renegocia sustancialmente. Lo que se evidencia aquí es la contradicción del proyecto subalternista que apunta Dipesh Chakrabarty en Provincializing Europe al ser forjado a través de los macro relatos emancipadores de la Ilustración, en especial las nociones de la nación y de ciudadano. En "Beyond Representation” (2001), Rabasa discute la escalada aunque incompleta descolonización de los estudios subalternos desde la Italia de 1930 hasta la época de los Zapatistas (1994 en adelante), pasando por la India de Guha hacia 1980 (201). Para Gramsci, argumenta Rabasa, el camino liberador para lo subalterno era posible dentro de la modernidad y las formas de conocimiento no modernas eran consideradas “folklóricas”. Luego, con el colectivo hindú, se pasa a depender mucho en el papel del historiador como el representante o mediador de las voces suprimidas. Rabasa sugiere entonces que los estudios subalternos deben crear un espacio abierto a múltiples epistemologías e iniciativas políticas: "Subaltern studies, therefore, would not pretend to have a priviledged access to subalterns but, rather, would define intellectual work as one more intervention in insurgent movements" (Rabasa, "Representation” 197). Esta teoría itinerante tiene una historia más densa de lo que Rabasa y Beverley toman en cuenta. El punto de partida no es simplemente Gramsci y la Italia hacia 1930 sino que se nutre de la experiencia colonial del sistema mundo moderno. Las observaciones de Gramsci sobre las políticas religiosas en Latinoamérica, los jesuitas en la India, el Islam y la modernidad, la religión y el estado en Japón le permiten entrever las dinámicas relaciones entre el estado, los intelectuales y las clases populares (Gramsci, “Religion”). 
Igualmente, nosotros mismos como intelectuales no podemos imaginarnos libres de tales problemáticas. No podemos asumirnos como sujetos del conocimiento plenamente coherentes y válidos. Es decir, se nos presentan problemas de doble filo que quizás no podamos rebasar (Spivak 171-73). ${ }^{12}$

Lo anterior, sin embargo, no descalifica las narrativas en cuestión, ni los proyectos por desmontar la violencia epistemológica. Podemos ver estas historias como un nativismo crítico, como el que propone Benita Parry, el cual surge de la necesidad de activar y renovar la memoria por parte de un sujeto descentrado y heterogéneo, no la simple conservación de la tradición por parte de un individuo autónomo fijo (173-74). Las narraciones Maya tzeltal recopiladas por Gómez Gutiérrez reelaboran el pasado para presentar la resistencia de Juan López al ejército colonial en 1712 en forma claramente relacionada con la rebelión del EZLN. En estas narraciones mayas encontramos una serie de mecanismos que reelaboran el pasado para crear un presente abierto a la negociación y la insurgencia. Entre estos mecanismos están la inversión de la historia, la compresión de diferentes hechos históricos, la invocación de poderes suprahumanos, la primitivización e incorporación del otro y una lectura profética del tiempo. Todas estas reelaboraciones del pasado permiten reconstruir una memoria maya en la cual la relación entre la sociedad dominante y las comunidades indígenas está lejos de ser, en forma definitiva, una de vencedores y vencidos y donde la insurgencia está latente.

\section{II. ¿Cómo LeER A JuAN LóPez? Los ESTUdios SUBALTERNOS Y LOS RETOS DE LA DESCOLONIZACIÓN} DE LA MEMORIA

En esta parte comenzaré contrastando la visión de esta rebelión maya que nos dan los relatos recopilados por Gómez Gutiérrez y la historiografía tradicional para resaltar los retos que nos presentan estas historias sobre la figura heroica y mítica de Juan López. Es decir, argumentaré que será preciso examinar bien los límites y legados coloniales de la historiografía occidental por su visión secular del tiempo, por asumir la noción occidental del sujeto y por seguir viendo las historias de países que otrora fueron colonias europeas bajo un marco de análisis de claro corte occidental, la nación. A cambio, es necesario abordar estas historias desde la perspectiva maya del tiempo y la memoria. Sólo entonces podremos ver estas historias mayas más que como relatos folklóricos o "literatura” oral (opuesta a la historia), como parte integral de los múltiples registros en los cuales se mueven las comunidades indígenas hoy-lucha armada, protestas, huelgas, litigio en ámbitos regionales, nacionales e internacionales_- para defender y negociar espacios ante las desigualdades geopolíticas que viene imponiendo la actual globalización.

\footnotetext{
${ }^{12}$ Gareth Williams con gran acierto problematiza la relación centro/periferia que reproducen los estudios subalternos. Pese a sus intenciones de crear una praxis intelectual no totalizadora y solidaria, sólo se logra crear un espacio de fantasía, en el sentido discutido por Slavoj Zizek, donde se afirma en última instancia la identidad estable del intelectual latinoamericanista sobre la diferencia radical del "otro" (231). Esta misma afirmación del centro como la vanguardia teórica y la producción de América Latina como una sub-alternidad, un espacio en blanco para ser explotado por los mercados teóricos transnacionales es lo que encuentra Moraña problemático de esta 'nueva' corriente teórica.
} 


\section{Vírgenes y naguales}

La rebelión de 1712 es conocida principalmente por la aparición de la virgen, Nuestra Señora del Rosario, a María de la Candelaria, una joven del pueblo de Cancuc en los Altos de Chiapas. Se dijo que la virgen había venido a ayudar a los indígenas y a liberarlos del yugo colonial. Esta es la convocatoria que circuló por la región:

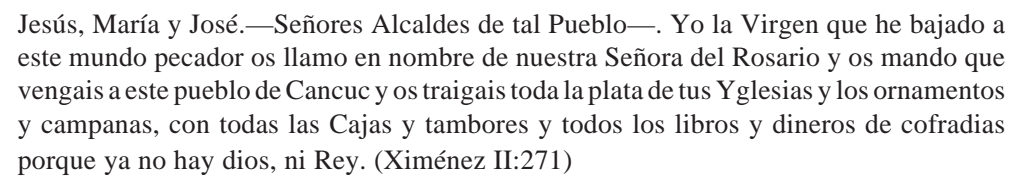

Según declararon los rebeldes, María de la Candelaria y otros líderes indígenas fueron instruidos por la virgen para ordenar sacerdotes, celebrar misa, oficiar otros rituales cristianos así como ordenar capitanes del ejército insurgente. La insurgencia, en todo caso, tiene una primera fase no militar donde se rechaza y excluye al colonizador mediante el secreto: "No publiquen este secreto" afirman los comunicados de Cancuc sobre la aparición de enviados a los pueblos de la región. ${ }^{13}$ Veinte y un pueblos tzeltales, tzotziles y choles respondieron al llamado de Cancuc y los que no se unieron a la rebelión recibieron represalias por parte de los auto-proclamados “soldados de la virgen”. Desde Ciudad Real, hoy San Bartolomé de las Casas, Guatemala y Tabasco fueron enviadas tropas del ejército español para la "pacificación” de la región. Seis meses de lucha armada culminaron con la toma de Cancuc por parte de las fuerzas españolas. El ejército español permaneció en la región durante varios meses juzgando a los insurgentes, varios de ellos descuartizados y decapitados en las plazas de Cancuc y otros pueblos rebeldes. Algunos líderes, incluyendo a María de la Candelaria, lograron escapar y los rumores de una nueva rebelión continuaron por muchos años y tienen eco hoy día en la rebelión del Ejército Zapatista de Liberación Nacional (EZLN).

Los relatos recopilados por Gómez Gutiérrez poco nos dicen de esta historia de la virgen. Se trata de ocho narraciones breves sobre uno de los insurgentes de 1712, Juan López, un indígena de Bachajón (incluyendo la "versión conjuntada” del compilador). Provienen de Bachajón, Chilón, Ocosingo y Cancuc; todos estos son pueblos que participaron en esa rebelión. Los relatos varían en algunos puntos, sobre todo en los detalles, aunque el nacimiento, hazañas, muerte y resurrección del personaje son una constante. En términos generales, Juan López nació en Bachajón. Fue hijo de una muchacha indígena virgen quien quedó embarazada al entrar en una cueva, un lugar sagrado en la cosmología maya. La familia y la comunidad se escandalizan cuando se enteran del estado de la joven, pero un “ángel” les revela su origen divino: su padre es un ajaw o dios de la naturaleza. Al nacer se ven obligados a llevar al niño a la cueva, donde desaparece hasta años más tarde, cuando regresa hecho hombre. Su retorno coincide con una incursión militar contra Cancuc. Los ancianos de Bachajón van a detener el ejército

\footnotetext{
${ }^{13}$ Papeles encontrados entre los rebeldes de Cancuc, traducidos al español. 14 de dic., 1712. AGI,
} Guatemala, legajo 294, folio 204. 
con sus poderes especiales, sus naguales. Juan López los alcanza y les dice que él solo puede detener el ejército. Los ancianos dudan de los poderes del joven pero éste los convence al producir un terremoto con su bastón. En Cancuc el ejército disparó contra Juan López hasta acabar con sus municiones. Entonces con su bastón mágico, convertido en fusil, Juan López logra destruir todo el ejército español. Luego, Juan López va a Oxchuc (tres nudos) donde es apresado por su propia gente en tres ocasiones distintas, pues siempre logra evadírseles. Tratan de deshacerse de él matándolo, decapitándolo y arrojándolo a una fosa. Pero, como Hunahpu e Ixbalanqué del Popol Vuh, Juan López regresa una y otra vez. Varios de los relatos afirman que recientemente se le ha visto de nuevo y que muy pronto regresará.

Al ver estas diferencias entre la historia de la virgen y la de Juan López, es claro que la memoria de esta rebelión está escindida en por lo menos dos vertientes. No se trata de dos versiones completamente aparte ni una exclusivamente nativa y la otra colonial, pues el culto a la virgen tiene claros elementos maya y la figura mítica de Juan López presenta elementos cristianos. El enigma es que en la documentación colonial, Juan López es una figura marginal. Fray Francisco Ximénez, una de las más recurridas fuentes para esta rebelión, lo menciona sólo de paso. López es descrito como un principal de Bachajón quien fue ahorcado por los insurgentes "por haber quitado a la Virgen lo que era suyo" (Ximénez II 285). ¿Hay acaso aquí una tensión entre los líderes marianistas y los nagualistas?

\section{El nagualismo, un discurso contra-hegemónico}

Los relatos tzeltales sobre Juan López elaboran un discurso radical a través del nagualismo. Presente en las culturas mesoamericanas desde tiempos prehispánicos, el nagualismo comprende una compleja serie de prácticas y creencias. El nagual o ch'ulel en Chiapas es el alter ego o el guardián de los mayas y los nahuatlecos, simbolizado en un animal o una fuerza natural: jaguares, coyotes, serpientes, pájaros, rayos, terremotos, etc. El nagualismo expresa una subjetividad extra somática donde las fronteras entre el sujeto y el mundo no están contrapuestas como sucede en el individuo autónomo de la modernidad occidental (el sujeto cartesiano). Desde la colonia, el nagualismo ha sido una práctica de resistencia a la autoridad colonial. Sociedades secretas clandestinamente practicaban rituales en las cuevas y las montañas, lejos de la mirada disciplinaria de las autoridades coloniales y eclesiásticas (Brinton, Nagualism 12). Kevin Gosner ve el nagualismo como una teoría indígena del poder, pues ha sido clave en la legitimación de las autoridades amerindias (Gosner 110). Este puede ser el caso de la rebelión de 1712. Sabemos por la visita que hizo fray Francisco Núñez de la Vega, obispo de Chiapas, unos años antes de la rebelión (1698) que el nagualismo era ampliamente practicado en la región (752-60). Juan Pablo Viqueira argumenta que la rebelión fue apoyada por dos sistemas religiosos opuestos, aunque también interpenetrados mutuamente y yuxtapuestos en la asimétrica relación colonial que los enmarca: por una parte, estaba el culto cristiano a la virgen y localizado en el pueblo. Por otra parte, estaba el culto indígena localizado en las cuevas, el nagualismo. Ninguno de los líderes del culto a la virgen era reconocido como 
nagualista. Sin embargo, si apelaron a sus curanderos y curanderas para que detuvieran al ejército español (Ximénez II 303). También en las confesiones y declaraciones oficiales se afirmó que los capitanes insurgentes fueron elegidos porque tenían naguales muy poderosos (Gosner 131). Los capitanes, sin embargo, estaban subordinados al clero indígena que controlaba el culto a la virgen desde Cancuc. La rebelión sería recordada por muchos años, según el historiador decimonónico Vicente Pineda, ya que se decía que los insurgentes habían escondido el tesoro recogido en la rebelión en las montañas cerca de Cancuc, probablemente como parte de las prácticas nagualistas (Pineda 68-9). El nagualismo es, con gran probabilidad, un elemento altamente significativo en la rebelión de 1712, en las formas cómo los insurgentes planearon y concibieron la rebelión. Es decir, siguiendo a Guha, si hemos de reconocer la agencia y conciencia indígena, es preciso tener en cuenta el nagualismo, un elemento que no ha considerado suficientemente la historiografía tradicional. Su persistencia no puede menospreciarse. En la rebelión de 1994, el nagualismo sigue siendo un elemento importante, el ch’ulel de Marcos, es una culebra gigante que espanta los soldados de la selva, según un relato de Marián Pérez Tzu, un tzotzil de San Cristobal. ${ }^{14}$

Las historias sobre Juan López se pueden entender mejor en relación al nagualismo. El hecho de que naciera, creciera y muriera (decapitado) en una cueva claramente lo inscribe como una deidad, un ajaw. Los relatos en cuestión señalan que estas cuevas se convirtieron en importantes centros ceremoniales tzeltzales (Gómez Gutiérrez 98,102, 107). Juan Méndez presenta a Juan López como un héroe que protege los tesoros escondidos en una cueva cerca de Cancuc (109). La centralidad de la cueva no ha de extrañarnos, ya que en el Popol Vuh y en otros relatos orales mayas, las cuevas son lugares que comunican con el mundo inferior, Xibalbá (Pickands 108).

Además de su relación con lugares asociados con el nagualismo, el poder de Juan López como nagualista es central en todas las historias. Hay viejos sabios que son poderosos nagualistas: gavilanes, tigres, caballos, zorrillos, arañas, rayos. Juan López los derrota a todos (Gómez Gutiérrez 83, 91, 97,101): "Realmente Juan López era de las personas con un poder sobrenatural, insuperable” afirma Miguel Vásquez en el primer relato (84).

Las historias recopiladas por Gómez Gutiérrez no son las únicas que registran el papel de los nagualistas en los conflictos étnicos en la región, donde se alude a hechos históricos que datan de años o acaso siglos atrás, procesados por una memoria de larga duración que los sintetiza a su manera. La colección de cuentos de Zinacantán, recopilada por Robert Laughlin, nos ofrece varios ejemplos. El relato de Romin Teratol narra cómo los ancianos se convirtieron en el rayo, el tornado y el sapo para destruir a los guatemaltecos y liberar los tzotziles que tenían en la prisión (21-26). Esta historia podría aludir al pasado colonial cuando Guatemala gobernaba a Chiapas. Otro relato de Manuel K’Obynox es más explícito al narrar los esfuerzos por detener el ejército que se envió desde Tabasco para reprimir la rebelión de 1712. Los nagualistas pusieron gusanos en la comida de los soldados, les enviaron tormentas y lluvia, pero no pudieron detenerlos. Trataron de

14 “Conversaciones ininterrumpidas: Las voces indígenas del mercado de San Cristóbal” traducidas del tzotzil por Jan Rus. 
seducirlos con mujeres y finalmente los destruyeron con una inundación (Laughlin 32830).

Si bien la figura de Juan López está más asociada con el nagualismo que con el culto a la virgen, también está estrecha y complejamente relacionada con el discurso cristiano. ¿Quién es Juan López? Un héroe tzeltal, según el subtítulo castellano. También se dice que es una figura similar a Cristo quien vive como gente común y corriente. Por otra parte, el subtítulo en tzeltal es Bats'il ajaw, literalmente “rey verdadero". Pero ajaw también es un ser supranatural: el padre de Juan López es un ajaw natural y el ángel que anunció el nacimiento del héroe también es un ajaw (118). La versión de Miguel Vázquez nos ilustra cómo hay dos códigos que están ambivalentemente presentes en esta figura, el cristiano y el maya:

Sabemos que su destino era ofrendar su vida por la libertad de su pueblo, pagar con su vida todos los males cometidos. No dejo de pensar en la vida de Cristo, que vino a otra tierra. Parece el cristo tzeltal, con características similares: sencillo, humilde, inofensivo. Me pregunto ¿Será nuestro Dios? Respondo que es un ajaw maya, un Salvador. No simplemente hombre poderoso, hombre inteligente, es un ajaw o Dios. (89)

Vásquez nos ofrece inicialmente una idea ambivalente del sacrificio (maya y cristiano). Luego señala la similitud con Jesucristo, en un giro que resalta el código ladino/ cristiano como el código principal para la interpretación de esta figura. Pero luego se desplaza hacia el código maya, enfatizando la diferencia entre Cristo y Juan López y reafirmando su divinidad en términos mayas: Ajaw Lum, Ajaw K’inal (90). Hay muchos paralelos con el nacimiento, los milagros y la muerte de Cristo, pero en cada caso hay algún elemento maya que reinscribe su historia en el mundo maya. Por ejemplo, nace de una virgen pero en una cueva, como anotamos arriba. También sus milagros son debidos a sus naguales. Y su muerte asemeja más el sacrificio maya o la figura del tramposo, que la de la crucifixión cristiana. Juan López es reiteradamente asociado con el sol (102, 104, 116, 129). Por lo tanto es muy probable que represente el Dios/Sol maya o J'ch'ul Totik.

Héroe y anti-héroe, personaje mítico e histórico a la vez, esta ambivalente figura se resiste a ser encasillada en las concepciones occidentales del tiempo y del sujeto. ¿Cómo, entonces, leer a Juan López? Más aún, podríamos preguntarnos ¿para qué leer a Juan López? Es decir, ¿qué nos puede decir este texto que no podamos sacar de cientos de folios en el Archivo General de Indias o de los numerosos textos históricos que han escudriñado minuciosamente fuentes documentales eclesiásticas, oficiales, económicas, etc.?

\section{Tiempo insurgente}

En Latinoamérica, las relaciones inter-étnicas coloniales han sido examinadas en gran parte a través de los conflictos, la resistencia y las rebeliones. Las rebeliones coloniales han generado, en palabras de una reconocida antropóloga, una "riqueza de información” (Bricker 5, mi traducción). Es cierto, las rebeliones coloniales pueden ser vistas como una “riqueza” documental (otro producto colonial). La rebelión tzeltal, tzotzil 
y chol en Chiapas en 1712 es un ejemplo representativo. Hay cuatro legajos en el Archivo General de Indias de unos seis mil folios, además de otros documentos que se encuentran en archivos regionales en Guatemala y México. ${ }^{15}$ La escritura no se detiene allí. La rebelión ocupa la atención de agentes coloniales y misioneros, historiadores, antropólogos y novelistas. Esta riqueza de información, sin embargo, aún simpatizando con la causa rebelde ha sido escrita en castellano e inglés, primordialmente para y por el régimen (neo)colonial.

Desde una perspectiva poscolonial que trata de cesar la violencia epistemológica es preciso preguntarse cómo estos textos sobre la rebelión representan, producen e interpelan el sujeto insurgente colonial. ¿En qué sentido puede decirse que estos textos son parte de la violencia colonial? Hay violencia en la historiografía cuando se suprime la conciencia histórica de los grupos subalternos, por ejemplo, como lo señala Guha en el artículo citado arriba. El caso de las guerras de Independencia es ilustrativo. Se presenta un pueblo que responde a figuras paternales y heroicas como Simón Bolívar, El libertador.

La práctica de la escritura sobre las rebeliones no sólo ha suprimido la conciencia subalterna, sino que también ha sido parte clave de un código de represión. Las narraciones producidas, bien señala Guha, cobran sentido primordialmente como un código contrainsurgente. Un claro ejemplo es la Historia de las sublevaciones indígenas habidas en el estado de Chiapas (1888) de Vicente Pineda:

Las barbaridades de la guerra de castas, que, cual bestia feroz, no distingue sexo, edad, ni condición en las personas, y que para satisfacer sus instintos salvajes, el bárbaro destruye é incendia cuanto encuentra a su paso, sorprendiendo y asesinando en las altas horas de la noche a moradores é inofensivos, nos impone el deber de dar á conocer á nuestros conciudadanos los principales hechos de armas habidos en las cuatro sublevaciones; las atrocidades y todo lo que pueda evitarles nuevas sorpresas para lo sucesivo. (9)

El reto para el crítico o historiador poscolonial es romper con esta "prosa contrainsurgente" con el fin de ver las rebeliones indígenas como una praxis deliberada y conciente que puede tener lógicas completamente diferentes de las de los proyectos anticoloniales y nacionalistas criollos. Esto es lo que me he propuesto hacer con la rebelión maya de 1712, revisando desde la documentación colonial hasta recientes novelas y narraciones contemporáneas vistas en relación al conflicto actual en Chiapas. Si bien José Rabasa y John Beverley han partido de las reflexiones de Guha para discutir rebeliones coloniales en México y Perú, respectivamente, siento que no han profundizado suficientemente en ninguna de las dos rebeliones aludidas para examinar la compleja y contradictoria praxis de la escritura de la insurgencia colonial. ¿Qué tipo de subjetividades se crean a través de la representación de la violencia? ¿Cómo escribir efectivamente contra

\footnotetext{
${ }^{15}$ Archivo General de Indias, Guatemala 293, 294, 295 y 296. Véase también Gosner.

${ }^{16}$ La representación de la violencia la trabajo con mayor profundidad en la parte dedicada a un drama sobre esta rebelión escrito por el antropólogo Daniel Brinton, a partir de la reflexiones críticas sobre la representación de la violencia discutidas por José Rabasa, Michael Taussig, Elaine Scarry y Gyanendra Pandey.
} 
la violencia sin reproducirla? ¿Como la violencia marca la memoria? Estas son algunas preguntas que estoy indagando en este proyecto sobre la poética insurgente. ${ }^{16}$

Lo que nos ofrecen los relatos maya sobre Juan López es la posibilidad de problematizar cómo los macro relatos de la modernidad y la nación, así como las concepciones occidentales del sujeto y del tiempo subalternizan esta memoria maya, un proceso del cual no estamos exentos. Por ejemplo, la Historia del padre Ximénez de 1720 inscribe no sólo esta rebelión, sino también las "antiguas historias del Quiché” (es decir el llamado Popol Vuh) dentro de la historia de Guatemala en un relato claramente protonacionalista. Otro ejemplo es la novela Oficio de tinieblas (1962) de Rosario Castellanos, la cual trata sobre la rebelión en San Juan Chamula, Chiapas, en 1867. Castellanos reubica la rebelión en el México posrevolucionario, cuando se están tratando de implementar las reformas agrarias que tanto prometió la Revolución. La novela agudamente señala el racismo, los prejuicios, la corrupción y la reticencia al cambio de los finqueros, los sacerdotes, las familias tradicionales y los políticos de Ciudad Real. Con esta novela uno ve por qué se frustraron los proyectos revolucionarios. Sin embargo, al inscribir la rebelión indígena dentro del macro relato de la nación, se está negando o subordinando la visión chamula de la rebelión. Ahora bien, cómo contar una historia que no se vea coartada por el macro relato de la nación supone una problemática que ha abordado la crítica subalternista reciente incluyendo a Partha Chatterjee, Gyan Pankash, Dipesh Chackrabarty, Florencia Mallón y John Beverley. Aunque me es imposible discutir con la amplitud necesaria los aportes de estos críticos a este debate, una preocupación central es cómo el modelo europeo de la nación como comunidad imaginada (homogénea, monolingüe, etc.) prefigura las historias de los países que otrora fueron colonias europeas. Es decir, si la concepción europea de la nación está prefigurando la historia de los países poscoloniales, ¿hasta qué punto puede hablarse de una historia poscolonial, esto es de una memoria descolonizada? La crítica subalternista ha propuesto entonces mirar aquellas voces que suprimió o subordinó el macro relato de la nación, sus fragmentos (Chatterjee) o las historias locales como historias nacionales alternas (Mallón) pero también historias que no necesariamente proyecten un relato nacional (Guha; Beverley).

Las historias mayas sobre Juan López son precisamente de este último tipo, ya que no proyectan ni se inscriben dentro de un macro relato nacional. Más bien están contrapuestas al relato de la nación, aunque no puede limitarse a un movimiento sólo de resistencia. ${ }^{17}$ El gobierno y los militares son percibidos como fuerzas externas que vienen a atacar a los pueblos maya. La figura de Juan López es parte de un discurso contrahegemónico el cual busca una autonomía o "respeto":

Es que los indios comenzaron a rebelarse, comenzaron a hacerse respetar. En nuestros días también sigue la rebeldía de los pueblos indios. Los tzeltales, junto con otros

\footnotetext{
${ }^{17}$ Como bien argumenta Brown en "Resisting Resistance” el enfocarnos en la resistencia puede ocultarnos otros aspectos claves de estas historias. El caso bien puede ser similar a la rebelión de los Asháninkas, un grupo amazónico en El Perú, en el cual las profecías jugaron un papel central: "The Asháninkas who inserted themselves into this conflict were not only responding to external challenge but also advancing their own vision of existential redefinition or transcendence" (Brown 731).
} 
indígenas, se levantaron el primer día del año de 1994 contra los malos tratos que sufren. El primer ejemplo fue el de Juan López en Bachajón. (120)

Esta memoria contra-hegemónica no se limita a las historias sobre Juan López. Victoria Bricker y Gary Gossen han estudiado varios dramas rituales en los cuales los mayas de los altos de Chiapas (tzeltales y tzotziles) expresan y refuerzan diferencias que en última instancia marcan estratégicamente los límites de su comunidad. El trabajo de Victoria Bricker sobre el sustrato mítico e histórico en los dramas mayas nos arroja luces sobre los relatos en cuestión. Bricker argumenta que la narrativa oral maya y los rituales que tratan sobre los conflictos étnicos parecen, en primera instancia, un bricolaje de eventos históricos. Es decir, aparentan ser una historia imperfecta. Por ejemplo, en los carnavales de Chamula, Chenalhó y Zinacantán, aparecen unas figuras extrañamente disfrazadas que representan a la vez a judíos, monos y soldados franceses. ¿Qué tipo de memoria pueden representar dichas figuras? Bricker sugiere que estos personajes tienen mucho sentido si tomamos la forma como opera la memoria maya. Simultáneamente, estas narraciones y rituales aluden a diferentes conflictos étnicos de diferentes periodos. La investigación documental provee el sustrato histórico de estas narraciones y rituales. En una profecía recitada en el carnaval de Chenalhó, por ejemplo, Bricker identifica siete hechos históricos diferentes, todos relacionados a conflictos étnicos: la crucifixión de Jesucristo, la guerra entre moros y cristianos, las campañas militares contra los indígenas Lacandones de los siglos XVI y XVII, la rebelión de 1712 discutida en este trabajo, la invasión francesa (1862-67), la rebelión de Chamula (1867-70) y el maltrato de los ladinos a los indígenas (135-36). ${ }^{18}$ Lo esencial es cómo se comprimen estos hechos históricos en una sola narración. Según Bricker, lo que importa no es su orden cronológico sino su valor estructural. Los judíos, monos, moros, soldados ocupan el lugar del otro. Es de igual modo que se presenta la oposición cristianos y judíos en la documentación sobre la rebelión de 1712, pero no es tan claro en las narraciones recopiladas por Gómez Gutiérrez. El esquema puede que no sea tan binario, como lo sugiere Gossen. En ello puede radicar la clave de cómo las comunidades mayas han logrado mantener cohesión a pesar de las invasiones de los mexicas, los españoles durante la colonia, las órdenes religiosas, las políticas estatales de aculturación y los desplazamientos forzados, pero también los trabajos migratorios y numerosos contactos nacionales y transnacionales (Collier 173). Se trata de la forma cómo las comunidades mayas de Chiapas estratégicamente regulan y seleccionan el contacto con el mundo percibido como "exterior", incluyendo otras comunidades mayas (vistas más allá del paradigma de las comunidades corporativas cerradas). La visión cíclica del tiempo permite una continua acomodación y compresión de nuevos actores y nuevas ideas

\footnotetext{
${ }^{18}$ Las identificaciones del "sustrato histórico" que hace Bricker son por lo general convincentes como probables referencias a diferentes hechos históricos. Otras interpretaciones, en cambio, son discutibles o necesitan un apoyo documental más contundente. Por ejemplo, Bricker sugiere que la figura Nana María Cocorina alude a María de la Candelaria, porque la palabra "nan” que significa madre también puede referirse a una virgen. A mi modo de ver, Gary Gossen ofrece una interpretación mucho más convincente. Las figuras de mujeres españolas en el carnaval de Chamula son presentadas como lujuriosas (126). Es un ritual que denigra a la madre de todos los ladinos, por lo tanto es un acto contestatario (127). La visión maya de la mujer, sin embargo, es mucho más compleja y plurifacética, como bien lo discute Gossen en el estudio citado.
} 
ubicándolos moralmente en el pasado (Gossen 25). De este modo los Chamulas primitivizan el otro. En las narraciones recopiladas por Gómez Gutiérrez sucede algo similar. Los soldados son presentados como salvajes (85). Esta visión cíclica del tiempo también permite traer el poder del pasado al presente para tornarlo en profecía, lo cual permite expresar, en términos de Bricker, una revitalización cultural. Esto es, en otras palabras, un poderoso nativismo maya que transforma el pasado.

\section{Subversiones del pasado}

En los relatos sobre Juan López, los militares que atacan a Cancuc son como los soldados mexicanos contemporáneos, descritos como "uniformados” (96), "soldados del gobierno” (82). Gómez Gutiérrez hace la conexión más explícita: "Pudo ser como ahora, que observamos en los últimos años que nuestros pueblos indígenas se encuentran cercados de soldados federales que se proponen capturar a supuestos alzados zapatistas" (121). Lo interesante son las numerosas subversiones simbólicas que efectúan los relatos. Por ejemplo, son los mayas los que establecen retenes, no el ejército (96). En las narraciones orales Juan López elimina al ejército en Cancuc, invirtiendo los roles de vencedores y vencidos, como en los carnavales. En el Festival de los Juegos de Chamula, se recrea ritualmente un presente en el cual los indígenas de este pueblo son los vencedores y sus enemigos (incluyendo los instintos propios) son desterrados (Gossen 102).

El pasado que surge en estas narraciones mayas es un pasado trasmutado, volcado al presente y tornado en profecía. Esta es la forma maya de concebir el tiempo. Se trata de una cultura que desde tiempos prehispánicos se ha caracterizado por sus calendarios y profecías. En el periodo clásico, los mayas registraban ciclos temporales de miles de años. Hoy día, en partes de Chiapas el calendario anual todavía se observa (Villa Rojas 149). Según Miguel León Portilla, el mundo maya esta básicamente elaborado a partir de una crono visión. El tiempo es sagrado: los glifos de los códices y los textos de procedencia maya y de redacción posterior a la conquista, han confirmado la continuada y obsesionante insistencia por conocer y prever la realidad siempre cambiante del kinh, sol, día, tiempo divinos” (León Portilla 59). El tiempo no es abstracto o un número matemático. Diferentes dioses encarnan el tiempo y son quienes tienen que cargarlo. La preocupación maya por el tiempo y las profecías son intentos de comprender la llegada y la partida de estos dioses que en última instancia rigen el destino de los humanos:

\footnotetext{
Los sacerdotes mayas se esfuerzan por medio de sus cómputos por conocer cuáles son esas presencias y cuál habrá de ser la resultante de varios influjos en determinados momentos. Como la naturaleza de kinh tiene por esencia ser cíclica, importa sobre todo conocer el pasado para entender el presente y predecir el futuro. (León Portilla 63)
}

En esencia, las narraciones mayas contemporáneas discutidas arriba así como los dramas rituales en la Chiapas de hoy día conllevan esta misma comprensión del tiempo. Aunque traten sobre el pasado, están marcadas por una preocupación por el futuro. El pasado se torna en modelo del presente y el futuro. Por esta razón, estas narraciones y rituales son claves en los movimientos de revitalización cultural. Esta tradición profética, 
según Bricker, constituye un “conscious cyclical revival of selected aspects of Maya culture, which is nativism” (180).

Bricker argumenta que varias de las rebeliones que han ocurrido en Chiapas, incluyendo la rebelión de 1712, fueron primero movimientos de revitalización cultural. Se convirtieron en resistencia armada cuando las autoridades coloniales y ladinas trataron de suprimir estos cultos locales. Quizás podemos invertir la tesis de Bricker para afirmar que la voluntad política, la cual incluye la rebelión, se expresa en términos religiosos o que hay una relación dialéctica entre los proyectos políticos y los movimientos de revitalización religiosa que conforman la praxis de la insurgencia subalterna. La importancia de esta inversión es que permite retener la importancia de las dimensiones simbólicas del proceso sin reducirlo a lo "cultural” y sin velar las dimensiones económicas y políticas de este conflicto colonial.

En síntesis, si bien tanto los elementos mayas como cristianos fueron importantes en la rebelión de 1712, se ha tendido a resaltar los elementos cristianos, la aparición de la virgen en Cancuc. ¿Qué sucedió con el otro lado? Esto es precisamente lo que nos revela las narraciones sobre Juan López, las cuales no ven el pasado como un objeto fijo sino como una memoria transformable, perforada y rehecha en violentos contextos coloniales, que habla el presente e ilumina el futuro. Vista más allá de la nostalgia, se trata de una memoria maya posconquista que es perpetuamente reelaborada, que en gran medida resiste la colonización, pero cuya visión no se agota en la resistencia: “Diles que regresaré algún día para ayudarlos nuevamente con sus problemas. Pero mientras iré a vivir donde nace el sol” (116). Este tiempo donde la insurgencia nunca cesa es sin duda clave en la lucha actual en Chiapas.

\section{Bibliografía}

Ahmad, Aijaz. “Teoría, política subalternidad y poscolonialidad” Entrevista de Robert P. Rodríguez-Morazzani y Cliff Simms. Pensar (en) los intersticios: Teoría y práctica de la crítica poscolonial. Santiago Castro Gómez, et al. Bogotá: Pensar, 1999. 111-30. Archivo General de Indias. Audiencia de Guatemala. Legajos 293, 294, 295 y 296.

Beverley, John. Subalternity and Representation. Durham: Duke University Press, 1999. Bricker, Victoria Reifer. The Indian Christ, The Indian King: The Historical Substrate of Maya Myth and Ritual. Austin: University of Texas Press, 1981.

Brinton, Daniel G. Maria Candelaria: An Historic Drama from American Aboriginal Life. Philadelphia: McKay, 1897.

Nagualism. Philadelphia: McKay, 1894.

Brown, Michael. “On Resisting Resistance”. American Anthropologist 98/4 (1996): 72949.

Castellanos, Rosario. Oficio de tinieblas. México: Joaquín Mortiz, 1962.

Chakrabarty, Dipesh. Provincializing Europe. Princeton: Princeton University Press, 2000.

Chatterjee, Partha. The Nation and Its Fragments. Princeton: Princeton University Press, 1993. 
Chaturvedi, Vinayak, ed. Mapping Subaltern Studies and the Postcolonial. Londres: Verso, 2000.

Collier, George. Planos de interacción del mundo tzotzil. México: Instituto Nacional Indigenista, 1976.

“Subaltern Studies in the Americas”. José Rabasa, Javier Sanjinés y Robert Carr, guest editors. Dispositio/n 19/46 (1994).

Frischmann, Donald. "New Mayan Theatre in Chiapas: Anthropology, Literacy and Social Drama”. Negotiating Performance. Diana Taylor y Juan Villegas, eds. Durham: Duke University Press, 1994. 213-38

García Canclini, Nestor. Culturas híbridas: estrategias para entrar y salir de la modernidad. México: Grijalbo, 1989.

Gómez Gutiérrez, Domingo. Jwan Lopes Bats’il Ajaw/ Juan López héroe Tzeltal. Letras Mayas Contemporáneas, IV. México: Instituto Nacional Indigenista, 1996.

Gosner, Kevin. Soldiers of the Virgin: The Moral Economy of a Colonial Maya Rebellion. Tucson: University of Arizona Press, 1992.

Gossen, Gary H. Telling Maya Tales: Tzotzil Identities in Modern Mexico. Nueva York: Routledge, 1999.

Gramsci, Antonio. “Religion: A Movement and an Ideology”. Further Selections from The Prision Notebooks. Derek Boothman, ed. Minneapolis: University of Minnesota Press, 1995. 1-137.

Guha, Ranajit. “The Prose of Counter-Insurgency”. Selected Subaltern Studies. R. Guha y Gayatri Chakravorty Spivak, eds. Nueva York: Oxford University Press, 1988. 45-84.

ed. A Subaltern Studies Reader, 1986-1995. Minneapolis: University of Minnesota Press, 1997.

Guha, Ranajit y Gayatri Chakravorty Spivak, eds. Selected Subaltern Studies. Nueva York: Oxford University Press, 1988.

“Latin American Subaltern Studies Group”. Founding Statement. Boundary 2 20/3 (1993): 1-11.

Laughlin, Robert M. Of Cabbages and Kings: Tales from Zinacantán. Washington,DC: Smithsonian Institution, 1977.

León Portilla, Miguel. Tiempo y realidad en el pensamiento maya. 3a. ed. México: UNAM, 1994. 119-67.

Lienhard, Martín. "Los callejones de la etnoficción ladina en el área maya (Yucatán, Guatemala, Chiapas)”. Nueva Revista de Filología Hispánica 35/2(1987): 549-70. "Voces marginadas y poder discursivo en América Latina”. Revista Iberoamericana 66/193 (2000): 785-98.

Lloyd, David. “Ethnic Cultures, Minority Discourse and the State”. Francis Barker et al Colonial Discourse/ Postcolonial Theory. Manchester: Manchester University Press, 1994. 221-38.

Mallón, Florencia. Peasant and Nation. Berkeley: University of California Press, 1995. "The Promise and Dilemma of Subaltern Studies: Perspectives from Latin American History”. American Historical Review 99 (1994): 1491-1515.

Martín Barbero, Jesús. De los medios a las mediaciones. México: Ediciones Gili, 1987. 
Marzal, Manuel. El sincretismo iberoamericano. Lima: Pontificia Universidad Católica del Perú, 1985.

Montemayor, Carlos. Chiapas, la rebelión indígena de México. México: Joaquin Mortiz, 1997.

Morales, Heberto. Jovel, serenata a la gente menuda. México: Porrúa, 1992.

Moraña, Mabel. "El boom del subalterno”. Revista de Crítica Cultural 14 (1997): 48-53.

Núñez de la Vega, Francisco. “Carta IX Pastoral”. Constituciones Diocesanas del Obispado de Chiapas. México: UNAM, 1988. 752-60.

Pandey, Gyanendra. "In Defense of the Fragment: Writing about Hindu-Muslim Riots in India Today”. A Subaltern Studies Reader: 1986-1995. Ranajit Guha, ed. Minneapolis: University of Minnesota Press, 1997. 1-33.

Parry, Benita. "Resistance Theory / Theorising Resistance or Two Cheers for Nativism". Colonial Discourse/Postcolonial Theory. Francis Barker et al. Manchester: Manchester University Press, 1994. 172-93.

Pérez Hernández, Manuel. "Vivencias de nuestra palabra: el resurgimiento de la cultura maya en Chiapas”. Escritores Indígenas Actuales II . Carlos Montemayor, ed. México: Consejo Nacional para la Cultura y las Artes, 1992. 83-102.

Pérez Tzu, Marián. “Conversaciones ininterrumpidas: Las voces indígenas del mercado de San Cristóbal”. Democracia en tierras indígenas: las elecciones en los Altos de Chiapas (1991-1998). Jan Rus, trad. Juan Pedro Viqueira y William Sonnleitner, coords. México: CIESAS/Colegio de México, 2000. 259-67.

Pickands, Martin. “The Hero Myth in Maya Folklore”. Symbol and Meaning beyond the Closed Community. Gary Gossen, ed. Albany: Institute for Mesoamerican Studies, 1986. 101-23.

Pineda, Vicente. Historia de las sublevaciones indígenas habidas en el estado de Chiapas México, 1888.

Rabasa, José. Writing Violence in the Northern Frontier. Durham: Duke University Press, 2000.

"Beyond Representation? The Impossibility of the Local”. The Latin American Subaltern Studies Reader. Durham: Duke University Press, 2001. 191-210.

Recinos, Adrián, ed. Popol Vuh. México: Fondo de Cultura Económica, 1995.

Rodríguez, Ileana, ed. The Latin American Subaltern Studies Reader. Durham: Duke University Press, 2001.

ed. Convergencia de tiempos: Estudios subalternos / contextos latinoamericanos, estado, cultura, subalternidad. Amsterdam: Rodopi, 2001.

Rowe, William y Vivian Schelling. Memory and Modernity: Popular Culture in Latin America. Londres: Verso, 1991.

Rus, Jan. "Conversciones ininterrumpidas: las voces indígenas del mercado de San Cristóbal”. Democracia en tierras indígenas: las elecciones en los Altos de Chiapas (1991-1998). Juan Pedro Viqueira y Willibald Sonnleitner, eds. México: CIESAS/ El Colegio de México, 2000. 259-67.

"Rereading Tzotzil Ethnography: Recent Scholarship from Chiapas, Mexico". Maya Culture and History in a Multicultural World. John Watanabe y Ed Fischer, eds. Albuquerque: School of American Research, en prensa. 
Scarry, Elaine. The Body in Pain. Nueva York: Oxford University Press, 1985.

Spivak, Gayatri Chakravorty. A Critique of Postcolonial Reason: Toward a History of a Vanishing Present. Cambridge: Harvard University Press, 1999.

Steele, Cynthia. "Indigenismo y posmodernidad: Narrativa indigenista, testimonio, teatro campesino y video en el Chiapas finisecular”. Revista de Crítica Literaria Latinoamericana 19/38 (1993): 249-60.

Taussig, Michael. Colonialism, Shamanism and the Wild Man: A Study in Terror and Healing. Chicago: The University of Chicago Press, 1987.

Viqueira, Juan Pedro. Indios rebeldes e idólatras: dos ensayos históricos sobre la rebelión india de Cancuc, Chiapas, acaecida el año de 1712. México: CIESAS, 1997.

Villa Rojas, Alfonso. "Los conceptos de espacio y tiempo entre los grupos mayances contemporáneos”. Miguel León Portilla. Tiempo y realidad en el pensamiento maya. México: UNAM, 1994. 119-67.

Williams, Gareth. "Fantasies of Cultural Exchange in Latin American Subaltern Studies”. The Real Thing: Testimonial Discourse in Latin America. Georg Gugelberger, ed. Durham: Duke University Press, 1996. 225-53.

Ximénez, Fray Francisco. Historia de la Provincia de San Vicente de Chiapa y Guatemala de la Orden de Predicadores. 3 vols. Guatemala: Biblioteca Goathemala, 1929-1931.

Zizek, Slavoj. The Sublime Object of Ideology. Londres: Verso, 1989. 